\title{
Certain numerical results in non-associative structures
}

\author{
Behnam Azizi ${ }^{1} \cdot$ Hossein Doostie ${ }^{1}$
}

Received: 12 September 2018 / Accepted: 20 December 2018 / Published online: 2 January 2019

(c) The Author(s) 2019

\begin{abstract}
The finite non-commutative and non-associative algebraic structures are indeed one of the special structures for their probabilistic results in some branches of mathematics. For a given integer $n \geq 2$, the $n$ th-commutativity degree of a finite algebraic structure $S$, denoted by $P_{n}(S)$, is the probability that for chosen randomly two elements $x$ and $y$ of $S$, the relator $x^{n} y=y x^{n}$ holds. This degree is specially a recognition tool in identifying such structures and studied for associative algebraic structures during the years. In this paper, we study the $n$ th-commutativity degree of two infinite classes of finite loops, which are non-commutative and non-associative. Also by deriving explicit expressions for $n$ th-commutativity degree of these loops, we will obtain best upper bounds for this probability.
\end{abstract}

Keywords Loop $\cdot$ Moufang loop $\cdot n$ th-commutativity degree

Mathematics Subject Classification $11 \mathrm{~B} 39 \cdot 20 \mathrm{P} 05 \cdot 20 \mathrm{~N} 05$

\section{Introduction}

Every algebraic structure here is non-commutative. A quasigroup is a non-empty set with a binary operation such that for every three elements $x, y$ and $z$ of that, the equation $x y=z$ has a unique solution in the set, whenever two of the three elements are specified. A quasi-group with a neutral element is called a loop, and following [5, 1-3], and one may see the definition of Moufang loop satisfying four tantamount relators. These loops are of interest because they retain main properties of the groups $[4,5]$. During the study of these loops an interesting class introduced by Chein [6-8] where, for a finite group $G$ and a new element $u,(u \notin G)$, the loop $M(G, 2)$ is defined as $M(G, 2)=G \cup G u$ such that the binary operation in $M(G, 2)$ is defined by:

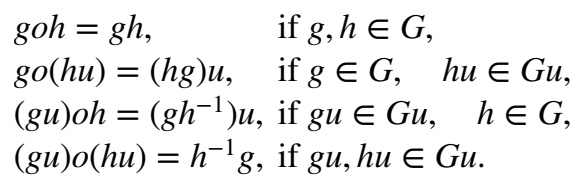

Hossein Doostie

doostieh@yahoo.com

Behnam Azizi

azizi_behnam396@yahoo.com

1 Department of Mathematics, Science and Research Branch, Islamic Azad University, P.O. Box 14515-1775, Tehran, Iran
These loops are studied for their finiteness property in [5]. It is obvious that $M(G, 2)$ is non-associative if and only if the group $G$ is non-abelian.

Following [9], recall the definition of $n$ th-commutativity degree of an algebraic structure $S$, denoted by $P_{n}(S)$, which is the probability that for two elements $x$ and $y$ of $S$ the relator $x^{n} y=y x^{n}$ holds, for a given integer $n \geq 2$. This degree has been studied mainly for associative algebraic structures during the years, one may consult Lescot [2], for example. We here intend to consider two classes of non-associative algebraic structures and calculate their $n$ th-commutativity degree, for every integer $n \geq 2$. Our considered classes of loops are $M\left(D_{2 m}, 2\right)$ and $M\left(Q_{2^{m}}, 2\right)$ where, for every $m \geq 3$, $D_{2 m}$ is the dihedral group of order $2 m$ and $Q_{2^{m}}$ is the generalized quaternion group of order $2^{m}$. Since these groups are non-abelian, the considered loops are non-associative loops. The $n$ th-commutativity degree of these loops will be calculated in "Main results" section, and the "Conclusion" section is devoted to study of upper bounds for the calculated probabilities.

To clear the terminology " $n$ th-commutativity" we have to mention the difference between this definition and that of Lescot [2] where, he used this terminology in the study of multiple commutativity degree of groups. 


\section{Main results}

Some preliminaries on groups are needed. Let $G$ be a finite group and $n \geq 2$ be a positive integer. We consider the $n$ thcommutativity set of $G$ as the following:

$C^{n}(G)=\left\{\left(g_{1}, g_{2}\right) \in G \times G \mid g_{1} g_{2}^{n}=g_{2}^{n} g_{1}\right\}$.

A simple computation shows that for every finite group $G$, $\left|C^{n}(G)\right| \geq 2|G|-1$. This set will be used in the computation of $P_{n}(G)=\frac{\left|C^{n}(G)\right|}{|G|^{2}}$. Also we use the presentations $<a, b \mid a^{m}=b^{2}=(a b)^{2}=1>$ $<a, b \mid a^{2^{m-1}}=1, \quad b^{2}=a^{2^{m-2}}, \quad(a b)^{2}=1>$ for the groups $D_{2 m}$ and $Q_{2^{m}}$, respectively. Our main results are:

Proposition 2.1 Let $G$ be a finite non-abelian group and $n \geq 2$ be a positive integer. If $M=M(G, 2)$ is a finite Moufang loop, then

$P_{n}(M)=\frac{1}{4}\left(P_{n}(G)+\frac{2 \alpha+\beta}{|G|}\right)$,

where $\alpha$ is the number of involutions of $G$ and $\beta$ is the number of elements $g \in G$ such that $g^{n}$ is involution.

Moreover, if $G$ be of odd order. Then

$P_{n}(M)=\frac{1}{4}\left(P_{n}(G)+\frac{3}{|G|}\right)$.

Two special cases of $G$ where $G=D_{2 m}$, the dihedral group of order $2 m$ and $G=Q_{2^{m}}$, the generalized quaternion group of order $2^{m}$, give us two infinite classes of Moufang loops $M\left(D_{2 m}, 2\right)$ and $M\left(Q_{2^{m}}, 2\right)$, where $m \geq 3$ is a positive integer. In fact, we prove the following results about these classes of loops:

Proposition 2.2 Let $M=M\left(D_{2 m}, 2\right)$ be a finite Moufang loop, where $m \geq 3$, and $n$ be an odd integer and $d=\operatorname{g.c} . d(m, 2 n)$. Then,

$P_{n}(M)= \begin{cases}\frac{3 m+d+5}{8 m}+\frac{1}{16 r}+\frac{1}{16}, & \text { m is odd, } \\ \frac{3 m+5}{8 m}+\frac{1}{8 r}+\frac{1}{16}, & \text { m is even, }\end{cases}$

where $r=\frac{m}{\text { g.c.d(m,n)}}$.

Proposition 2.3 Let $M=M\left(Q_{2^{m}}, 2\right)$ be a finite Moufang loop, where $m \geq 3$, and $n$ be an integer and $d=\operatorname{g.c} . d\left(2^{m-1}, 2 n\right)$. Then,

$P_{n}(M)= \begin{cases}\frac{7+d}{2^{m+2}}+\frac{1}{16}, & n \text { is odd }, \\ \frac{4+d}{2^{m+2}}+\frac{3}{8}, & n \equiv 0\left(\bmod 2^{m-2}\right), \\ \frac{4+d}{2^{m+2}}+\frac{1}{2^{r+4}}+\frac{5}{16}, & n \equiv \pm 2^{r}\left(\bmod 2^{m-2}\right),\end{cases}$ where $r=1,2, \ldots, m-3$.

At first, we give certain preliminary results as in the following lemmas:

Lemma 2.4 Let $D_{2 m}$ be a dihedral group, where $m \geq 3$, and $n$ be an odd integer, $d=$ g.c.d $(m, n)$ and $r=\frac{m}{d}$. Then

$\left|C^{n}\left(D_{2 m}\right)\right|= \begin{cases}m^{2}+2 m+\frac{m^{2}}{r}, & \text { mis odd }, \\ m^{2}+2\left(2 m+\frac{m^{2}}{r}\right), & \text { m is even. }\end{cases}$

Proof Let $m$ be an odd integer and we know that $D_{2 m}=A \cup B$, where $A=\left\{1, a, \ldots, a^{m-1}\right\}$ and $B=\left\{b, a b, \ldots, a^{m-1} b\right\}$. Clearly $\left|A^{n}\right|=\frac{|A|}{d}=\frac{m}{d}=r$, and the element $1 \in A^{n}$ commutes with all the elements of $D_{2 m}$ and each other element of $A^{n}$ commutes with all the elements $1, a, \ldots, a^{m-1} \in D_{2 m}$. This implies that there are $d \times(2 m+(r-1) m)=m^{2}+\frac{m^{2}}{r}$ pairs as $(a, g) \in A \times D_{2 m}$ such that $a^{n} g=g a^{n}$. Also $B^{n}=\left\{\left(a^{j} b\right)^{n} \mid a^{j} b \in B, \quad 0 \leq j \leq m-1\right\}=B$ and each element of $B$ commutes with two elements 1 and itself. So there are $2 m$ pairs as $(b, g) \in B \times D_{2 m}$ such that $b^{n} g=g b^{n}$. Consequently,

$\left|C^{n}\left(D_{2 m}\right)\right|=2 m+m^{2}+\frac{m^{2}}{r}$.

Now suppose that $m$ be an even integer. Then, $\left|A^{n}\right|=\frac{|A|}{d}=\frac{m}{d}=r$ and the elements $1, a^{r} \in A^{n}$ are central elements. Also, other elements of $A^{n}$ commute with all of the elements $1, a, \ldots, a^{m-1} \in D_{2 m}$. Thus there are $d(2(2 m)+m(r-2))=m^{2}+2 \frac{m^{2}}{r}$ pairs as $(a, g) \in A \times D_{2 m}$ such that $a^{n} g=g a^{n}$. In addition, $B^{n}=B$ and each element $a^{i} b(0 \leq i \leq m-1)$ of the set $B$ commutes with the four elements $\left\{1, a^{\frac{m-2}{2}}, a^{i} b, a^{j} b\right\}$, where $0 \leq i, j \leq m-1$ and $|i-j|=\frac{m}{2}$. So there are $4 m$ pairs as $(b, g) \in B \times D_{2 m}$ such that $b^{n} g \stackrel{2}{=} g b^{n}$. So we get

$\left|C^{n}\left(D_{2 m}\right)\right|=4 m+m^{2}+\frac{2 m^{2}}{r}$.

Lemma 2.5 Let $D_{2 m}$ be a dihedral group, where $m \geq 3$, and $n$ be an even integer, $d=$ g.c.d $(m, n)$ and $r=\frac{m}{d}$. Then,

$\left|C^{n}\left(D_{2 m}\right)\right|= \begin{cases}3 m^{2}+\frac{2 m^{2}}{r}, & r \text { is even }, \\ 3 m^{2}+\frac{m^{2}}{r}, & r \text { is odd } .\end{cases}$

Proof Let $r$ be an even integer and we know that $D_{2 m}=A \cup B$. Clearly $\left|A^{n}\right|=\frac{|A|}{d}=\frac{m}{d}=r$ and the 
elements $1, a^{r} \in A^{n}$ commute with all the elements of $D_{2 m}$ and each other element of $A^{n}$ commutes with the elements $1, a, \ldots, a^{m-1} \in D_{2 m}$. This implies that there are $d \times(2(2 m)+(r-2) m)=m \frac{m}{r}(r+2)$ pairs as $(a, g) \in A \times D_{2 m}$ such that $a^{n} g=g a^{r}$. Also $B^{n}=\left\{\left(a^{j} b\right)^{n} \mid a^{j} b \in B, \quad 0 \leq j \leq m-1\right\}=B$ and each element of $B$ commutes with all elements of $D_{2 m}$. So there are $2 m|B|=2 m^{2}$ pairs as $(b, g) \in B \times D_{2 m}$ such that $b^{n} g=g b^{n}$. Consequently,

$\left|C^{n}\left(D_{2 m}\right)\right|=2 m^{2}+m \frac{m}{r}(r+2)=3 m^{2}+\frac{2 m^{2}}{r}$.

The proof is similar, when $r$ be an odd integer.

Lemma 2.6 Let $Q_{2^{m}}$ be a generalized quaternion group, where $m \geq 3$, and $n$ be an odd integer. Then,

$\left|C^{n}\left(Q_{2^{m}}\right)\right|=2^{m}\left(3+2^{m-2}\right)$.

Proof Let $n$ be an odd integer and note that $Q_{2^{m}}=A \cup B$, where $A=\left\{1, a, \ldots, a^{2^{m-1}-1}\right\}$ and $B=\left\{b, a b, \ldots, a^{2^{m-1}-1} b\right\}$. Clearly $A^{n}=\left\{a^{j n} \mid a^{j} \in A, \quad 0 \leq j \leq 2^{m-1}-1\right\}=A$. Obviously the elements $1, a^{2^{m-2}} \in A^{n}$ are central elements in $Q_{2^{m}}$ and each other element of $A^{n}$ commutes with all the elements $1, a, \ldots, a^{2^{m-1}} \in Q_{2^{m}}$. So there are $2\left(2^{m}\right)+\left(2^{m-1}-2\right) 2^{m-1}=2^{m}\left(1+2^{m-2}\right)$ pairs as $(a, g) \in A \times Q_{2^{m}}$ such that $a^{n} g=g a^{n}$. Also $B^{n}=B$ and each element of $B$ commutes with the four elements in $Q_{2^{m}}$. This shows that there are $4\left(2^{m-1}\right)$ pairs as $(a, g) \in B \times Q_{2^{m}}$ such that $b^{n} g=g b^{n}$. Hence,

$\left|C^{n}\left(Q_{2^{m}}\right)\right|=2^{m}\left(1+2^{m-2}\right)+4\left(2^{m-1}\right)=2^{m}\left(3+2^{m-2}\right)$.

Lemma 2.7 Let $Q_{2^{m}}$ be a generalized quaternion group, where $m \geq 3$, and $n$ be an even integer. Then,

$\left|C^{n}\left(Q_{2^{m}}\right)\right|= \begin{cases}2^{2 m}, & n \equiv 0 \quad\left(\bmod 2^{m-2}\right), \\ 3\left(2^{2 m-2}\right)+2^{2 m-r-2}, & n \equiv \pm 2^{r} \quad\left(\bmod 2^{m-2}\right),\end{cases}$

where $r=1,2, \ldots, m-3$.

Proof If $n \equiv 0\left(\bmod 2^{m-2}\right)$, then $Q_{2^{m}}^{n}=\left\{g^{n} \mid g \in Q_{2^{m}}\right\}=\{1\}$. So,

$\left|C^{n}\left(Q_{2^{m}}\right)\right|=2^{m} 2^{m}=2^{2 m}$.

Now suppose that $n \equiv \pm 2^{r}\left(\bmod 2^{m-2}\right)$. We know that $Q_{2^{m}}=A \cup B$. Clearly $d=$ g.c.d $\left(2^{m-1}, n\right)=\frac{2^{m}}{2^{r+2}} \quad$ and $\left|A^{n}\right|=\frac{|A|}{d}=2^{r+1}$. Also the elements $1, a^{2^{r}} \in A^{n}$ are central elements in $Q_{2^{m}}$ and each other element of $A^{n}$ commutes with all the elements $1, a, \ldots, a^{2^{m-1}-1} \in Q_{2^{m}}$. So there are $d \times\left(2\left(2^{m}\right)+\left(2^{r+1}-2\right) 2^{m-1}\right)=2^{2 m-r-2}+2^{2 m-2}$ pairs as $(a, g) \in A \times Q_{2^{m}}$ such that $a^{n} g=g a^{n}$. On the other hand, for every $0 \leq j \leq 2^{m-1}$ we have $\left(a^{j} b\right)^{n}=b^{n}$ and $b^{n}$ is equal to 1 or $a^{2^{m-2}}$, and these elements are central elements in $Q_{2^{m}}$. So there are $2^{m-1} 2^{m}$ pairs as $(b, g) \in B \times Q_{2^{m}}$ such that $b^{n} g=g b^{n}$. So we get

$\left|C^{n}\left(Q_{2^{m}}\right)\right|=2^{2 m-r-2}+2^{2 m-2}+2^{m-1} 2^{m}=3\left(2^{2 m-2}\right)+2^{2 m-r-2}$.

By results of these lemmas, we get explicit values for $P_{n}\left(D_{2 m}\right)$ and $P_{n}\left(Q_{2^{m}}\right)$ as in the following:

Lemma 2.8 Let $D_{2 m}$ be a dihedral group, where $m \geq 3$, and $n$ be an odd integer, $d=\operatorname{g.c} . d(m, n)$ and $r=\frac{m}{d}$. Then,

$P_{n}\left(D_{2 m}\right)= \begin{cases}\frac{1}{4}+\frac{1}{2 m}+\frac{1}{4 r}, & \text { mis odd }, \\ \frac{1}{4}+2\left(\frac{1}{2 m}+\frac{1}{4 r}\right), & \text { mis even. }\end{cases}$

Proof By Lemma 2.4, and by definition of $P_{n}\left(D_{2 m}\right)=\frac{\left|C^{n}\left(D_{2 m}\right)\right|}{(2 m)^{2}}$, we deduce that

$P_{n}\left(D_{2 m}\right)= \begin{cases}\frac{m^{2}+2 m+\frac{m^{2}}{r}}{4 m^{2}}=\frac{1}{4}+\frac{1}{2 m}+\frac{1}{4 r}, & \text { mis odd, } \\ \frac{m^{2}+2\left(2 m+\frac{m^{2}}{r}\right)}{4 m^{2}}=\frac{1}{4}+2\left(\frac{1}{2 m}+\frac{1}{4 r}\right), & \text { mis even, }\end{cases}$

and the proof is complete.

Lemma 2.9 Let $D_{2 m}$ be a dihedral group, where $m \geq 3$, and $n$ be an even integer, $d=\operatorname{g.c} . d(m, n)$ and $r=\frac{m}{d}$. Then,

$P_{n}\left(D_{2 m}\right)= \begin{cases}\frac{3}{4}+\frac{1}{2 r}, & \text { ris even }, \\ \frac{3}{4}+\frac{1}{4 r}, & \text { ris odd } .\end{cases}$

Proof By Lemma 2.5, and by definition of $P_{n}\left(D_{2 m}\right)=\frac{\left|C^{n}\left(D_{2 m}\right)\right|}{(2 m)^{2}}$, we get the result

$P_{n}\left(D_{2 m}\right)= \begin{cases}\frac{3 m^{2}+\frac{2 m^{2}}{r}}{4 m^{2}}=\frac{3}{4}+\frac{1}{2 r}, & \text { ris even, } \\ \frac{3 m^{2}+\frac{m^{2}}{r}}{4 m^{2}}=\frac{3}{4}+\frac{1}{4 r}, & \text { ris odd },\end{cases}$

and the proof is complete. 
Lemma 2.10 Let $Q_{2^{m}}$ be a generalized quaternion group, where $m \geq 3$, and $n$ be an odd integer. Then,

$P_{n}\left(Q_{2^{m}}\right)=\frac{3+2^{m-2}}{2^{m}}$.

Proof By Lemma 2.6, and by definition of $P_{n}\left(Q_{2^{m}}\right)=\frac{\mid C^{n}\left(Q_{\left.2^{m}\right) \mid}\right.}{\left(2^{m}\right)^{2}}$, we deduce that

$P_{n}\left(Q_{2^{m}}\right)=\frac{2^{m}\left(3+2^{m-2}\right)}{2^{2 m}}=\frac{3+2^{m-2}}{2^{m}}$.

Lemma 2.11 Let $Q_{2^{m}}$ be a generalized quaternion group, where $m \geq 3$, and $n$ be an even integer. Then,

$P_{n}\left(Q_{2^{m}}\right)= \begin{cases}1, & n \equiv 0 \quad\left(\bmod 2^{m-2}\right), \\ \frac{3}{4}+\frac{1}{2^{r+2}}, & n \equiv \pm 2^{r} \quad\left(\bmod 2^{m-2}\right),\end{cases}$

where $r=1,2, \ldots, m-3$.

Proof By Lemma 2.7, and the definition of $P_{n}\left(Q_{2^{m}}\right)=\frac{\mid C^{n}\left(Q_{\left.2^{m}\right) \mid}\right.}{2^{2 m}}$, we get the result

$P_{n}\left(Q_{2^{m}}\right)= \begin{cases}\frac{2^{2 m}}{2^{2 m}}=1, & n \equiv 0\left(\bmod 2^{m-2}\right), \\ \frac{3\left(2^{2 m-2}\right)+2^{2 m-r-2}}{2^{2 m}}=\frac{3}{4}+\frac{1}{2^{r+2}}, & n \equiv \pm 2^{r}\left(\bmod 2^{m-2}\right),\end{cases}$

where $r=1,2, \ldots, m-3$.

To prove the main propositions, we recall the following properties of the Moufang loop $M=M(G, 2)$, where $G$ is a given group. These may be verified easily by considering the binary operation in $M$ :

(i) $\quad(g u) o h^{n}=h^{n} o(g u)$ if and only if $h^{2 n}=1$;

(ii) $(g u)^{n} o h=h o(g u)^{n}$ if and only if $h^{2}=1$;

(iii) $(g u)^{n} o(h u)=(h u) o(g u)^{n}$ if and only if $\left(g^{-1} h\right)^{2}=1$;

(iv) if $g^{n} h=h g^{n}$ then $(g u)^{n} o(h u)=(h u) o(g u)^{n}$ if and only if $h^{2}=g^{2}$.

Proof of Proposition 2.1 Le t $T=\left\{(g, h) \mid g, h \in M, g h^{n}=h^{n} g\right\}$. Simplify the binary operation $o$ in $M$ as the table

\begin{tabular}{lll}
\hline $\mathrm{o}$ & $\mathrm{G}$ & $\mathrm{Gu}$ \\
$\mathrm{G}$ & $\mathrm{G} * \mathrm{G}$ & $\mathrm{G} * \mathrm{Gu}$ \\
$\mathrm{Gu}$ & $\mathrm{Gu} * \mathrm{G}$ & $\mathrm{Gu} * \mathrm{Gu}$ \\
\hline
\end{tabular}

Since $P_{n}(M)=\frac{|T|}{(2|G|)^{2}}$, then it is sufficient to enumerate $|T|$. For every pair $(g, h) \in M$, there are four cases:

Case 1: $g, h \in G$. In this case, there are $\left|C_{n}(G)\right|$ number of elements of the type $(g, h)$ in $T$.

Case 2: $g \in G u$ and $h \in G$. Then, $g=g_{1} u, g_{1} \in G$. By (ii), we conclude that $h^{2}=1$. So, there are exactly $\alpha|G u|=\alpha|G|$ number of elements in $T$ of the type $\left(g_{1} u, h\right)$.

Case 3: $\quad g \in G$ and $h \in G u$. So, $h=h_{1} u$ where $h_{1} \in G$. In this case, we use ( $i)$ and deduce that there are $\beta|G|$ number of distinct elements in $T$ of the type $\left(g, h_{1} u\right)$, because of the validity of the relation $h_{1}^{2 n}=1$.

Case 4: $\quad g \in G u$ and $h \in G u$. So, $g=g_{1} u$ and $h=h_{1} u$, for $g_{1}, h_{1} \in G$. By using (iii), we confirm the existence of $\alpha|G|$ number of distinct elements in $T$ of the type $\left(g_{1} u, h_{1} u\right)$, for, $\left(g_{1}^{-1} h_{1}\right)^{2}=1$.

Consequently, $T$ has contains $\left|C^{n}(G)\right|+2 \alpha|G|+\beta|G|$ number of elements all together. Hence,

$\left.P_{n}(M)=\frac{\left|C^{n}(G)\right|+2 \alpha|G|+\beta|G|}{4|G|^{2}}\right)=\frac{1}{4}\left(P_{n}(G)+\frac{(2 \alpha+\beta)}{|G|}\right)$.

Now if $G$ be a finite group of odd order. Then, $\alpha=\beta=1$, and so

$\left.P_{n}(M)=\frac{\left|C^{n}(G)\right|+3|G|}{4|G|^{2}}\right)=\frac{1}{4}\left(P_{n}(G)+\frac{3}{|G|}\right)$.

Proof of Proposition 2.2 By Lemma 2.8, and Proposition 2.1, we get

$P_{n}(M)=\frac{1}{4}\left(P_{n}\left(D_{2 m}\right)+\frac{2 \alpha+\beta}{|G|}\right)$.

Moreover,

$\beta=\left\{\begin{array}{l}m+d, \text { mis even, } \\ m+1, \text { mis odd },\end{array}\right.$

and

$\alpha=\left\{\begin{array}{l}m+2, \text { mis even }, \\ m+1, \text { mis odd } .\end{array}\right.$ 
where $d=(m, 2 n)$. So,

$P_{n}(M) \leq \frac{11}{32}$,

$P_{n}(M)= \begin{cases}\frac{1}{4}\left(\frac{1}{4}+\frac{1}{2 m}+\frac{1}{4 r}+\frac{2(m+2)+(m+d)}{2 m}\right)=\frac{3 m+d+5}{8 m}+\frac{1}{16 r}+\frac{1}{16}, & \text { miseven, } \\ \frac{1}{4}\left(\frac{1}{4}+2\left(\frac{1}{2 m}+\frac{1}{4 r}\right)+\frac{3(m+1)}{2 m}\right)=\frac{3 m+5}{8 m}+\frac{1}{8 r}+\frac{1}{16}, & \text { mis odd. }\end{cases}$

where, $r=\frac{m}{g . c . d(m, n)}$ and the proof is complete.

Proof of Proposition 2.3 By Proposition 2.1,

$P_{n}(M)=\frac{1}{4}\left(P_{n}\left(Q_{2^{m}}\right)+\frac{2 \alpha+\beta}{|G|}\right)$.

Since $d=$ g.c. $d\left(2^{m-1}, 2 n\right)$, for odd values of $n, \alpha=2$, and $\beta=d$. In this case, Lemma 2.10 gives us:

$P_{n}(M)=\frac{1}{4}\left(\frac{3+2^{m-2}}{2^{m}}+\frac{4+d}{2^{m}}\right)=\frac{7+d}{2^{m+2}}+\frac{1}{16}$.

However, for even values of $n, \alpha=2$ and $\beta=d+2^{m-1}$. In this case, we use Lemma 2.11 and get, where $M=M\left(Q_{2^{m}}, 2\right)$ and $m \geq 3$ is an integer.

Proof For $n=p_{1}^{\alpha_{1}} p_{2}^{\alpha_{2}} \ldots p_{k}^{\alpha_{k}}$, we use Remark 3.1-(i) to get $d=\operatorname{g.c} . d\left(2^{m-1}, 2 n\right)=2$. So,

$P_{n}(M) \leq \frac{1}{32}(9+d)=\frac{1}{32}(9+2)=\frac{11}{32}$.

Acknowledgements The authors would like to thank the referees for a very careful reading of the paper and complete comments and useful suggestions, which improved considerably the presentation of this paper.

$P_{n}(M)= \begin{cases}\frac{1}{4}\left(1+\frac{4+d+2^{m-1}}{2^{m}}\right)=\frac{4+d}{2^{m+2}}+\frac{3}{8}, & n \equiv 0\left(\bmod 2^{m-2}\right), \\ \frac{1}{4}\left(\frac{3}{4}+\frac{1}{2^{r+2}}+\frac{4+d+2^{m-1}}{2^{m}}\right)=\frac{4+d}{2^{m+2}}+\frac{1}{2^{r+4}}+\frac{5}{16}, & n \equiv \pm 2^{r}\left(\bmod 2^{m-2}\right),\end{cases}$

where $r=1,2, \ldots, m-3$. This completes the proof.

\section{Conclusion}

The following remarks are the results of Proposition 2.3. They give us useful upper bounds for $P_{n}(M)$ where $M=M\left(Q_{2^{m}}, 2\right)$.

Remark 3.1 For all integers, $n \geq 2$ and $m \geq 3$ let $M=M\left(Q_{2^{m}}, 2\right)$ and $d=$ g.c.d $\left(2^{m-1}, 2 n\right)$. Then,

(i) For odd values of $n, P_{n}(M) \leq \frac{1}{32}(9+d)$,

(ii) For even values of $n$,

$$
\left\{\begin{array}{l}
P_{n}(M) \leq \frac{1}{32}(16+d), \quad n \equiv 0\left(\bmod 2^{m-2}\right), \\
P_{n}(M) \leq \frac{1}{32}(15+d), \quad n \equiv \pm 2^{r}\left(\bmod 2^{m-2}\right), r=1,2, \ldots, m-3
\end{array}\right.
$$

Remark 3.2 For distinct odd primes $p_{1}, \ldots, p_{k}$ and positive integers $\alpha_{1}, \ldots, \alpha_{k}$, if $n=p_{1}^{\alpha_{1}} p_{2}^{\alpha_{2}} \ldots p_{k}^{\alpha_{k}}$, then
Open Access This article is distributed under the terms of the Creative Commons Attribution 4.0 International License (http://creativeco mmons.org/licenses/by/4.0/), which permits unrestricted use, distribution, and reproduction in any medium, provided you give appropriate credit to the original author(s) and the source, provide a link to the Creative Commons license, and indicate if changes were made.

\section{References}

1. Ahmadidelir, K.: On the commutativity degree in finite Moufang loops. Int. J. Group Theory 5, 37-47 (2016)

2. Ahmadidelir, K.: On the non-commuting graph in finite Moufang loops. J. Algebra Appl. 17(4), 1850070 (2018). https://doi. org/10.1142/S02194988085007060

3. Chein, O.: Moufang loops of small order I. Trans. Am. Math. Soc. 188, 31-51 (1974)

4. Chein, O.: Moufang loops of small order. Mem. Am. Math. Soc. 13(197), 1-131 (1978)

5. Chein, O., Rajah, A.: Possible orders of non-associative Moufang loops. Comment. Math. Univ. Carol. 41, 237-244 (2000)

6. M. Hashemi., M. Polkouei.: Some numerical results on two classes of finite groups. J. Algebra and Realted Topics, 3 (1) (2015), 63-72. 
7. Goodaire, E.G., May, S., Raman, M.: The Moufang loops of order less than 64. Nova Science Publishers, Hauppauge (1999)

8. Lescot, P.: Isoclinism classes and commutativity degrees of finite groups. J. Algebra. 177, 847-869 (1995)
9. Naghy, G.P., Vojtěchovský, P.: The Moufang loops of order 64 and 81. J. Symb. Comput. 42(9), 871-883 (2007)

Publisher's Note Springer Nature remains neutral with regard to jurisdictional claims in published maps and institutional affiliations. 